\title{
Reprogramming fibroblasts into induced pluripotent stem cells with Bmi1
}

Jai-Hee Moon ${ }^{1}$, June Seok Heo ${ }^{1}$, Jun Sung Kim ${ }^{1}$, Eun Kyoung Jun ${ }^{1,2}$, Jung Han Lee ${ }^{1,2}$, Aeree Kim³, Jonggun Kim ${ }^{4}$, Kwang Youn Whang ${ }^{4}$, Yong-Kook Kang, Seungeun Yeo, Hee-Joung Lim ${ }^{4}$, Dong Wook Han ${ }^{6}$, Dong-Wook Kim ${ }^{7}$, Sejong $\mathrm{Oh}^{8}$, Byung Sun Yoon ${ }^{1}$, Hans R Schöler ${ }^{9}, 10$, Seungkwon You ${ }^{1}$

${ }^{I}$ Laboratory of Cell Function Regulation, College of Life Sciences and Biotechnology, Korea University, Seoul 136-701, Republic of Korea; ${ }^{2}$ Division of Stem Cell Research Institute, Stemmedience Corp., Seoul, Republic of Korea; ${ }^{3}$ Department of Pathology, College of Medicine, Korea University Guro Hospital, Seoul, Republic of Korea; ${ }^{4}$ Division of Biotechnology, College of Life Sciences and Biotechnology, Korea University, Seoul, Republic of Korea; ${ }^{5}$ Development and Differentiation Research Center, KRIBB, Daejeon 305-333, Republic of Korea; ${ }^{6}$ Department of Stem Cell Biology, SMART Institute of Advanced Biomedical Science, Konkuk University, 1 Hwayang-dong, Gwangjin-gu, Seoul 143-701, Republic of Korea ${ }^{7}$ Department of Physiology, Center for Cell Therapy, Yonsei University College of Medicine, Seoul, Republic of Korea, ${ }^{8}$ Division of Animal Science, Chonnam National University, Gwangju 500-757, Republic of Korea; ${ }^{9}$ Department of Cell and Developmental Biology, Max Planck Institute for Molecular Biomedicine,Röntgenstraße 20, Münster D-48149, Germany; ${ }^{10}$ Medical Faculty, University of Münster, Domagkstr. 3, Münster D-48149, Germany

Somatic cells can be reprogrammed into induced pluripotent stem (iPS) cells by the transcription factors Oct4, Sox2, and Klf4 in combination with c-Myc. Recently, Sox2 plus Oct4 was shown to reprogram fibroblasts and Oct4 alone was able to reprogram mouse and human neural stem cells (NSCs) into iPS cells. Here, we report that Bmi1 leads to the transdifferentiation of mouse fibroblasts into NSC-like cells, and, in combination with Oct4, can replace Sox2, Klf4 and c-Myc during the reprogramming of fibroblasts into iPS cells. Furthermore, activation of sonic hedgehog signaling (by Shh, purmorphamine, or oxysterol) compensates for the effects of Bmi1, and, in combination with Oct4, reprograms mouse embryonic and adult fibroblasts into iPS cells. One- and two-factor iPS cells are similar to mouse embryonic stem cells in their global gene expression profile, epigenetic status, and in vitro and in vivo differentiation into all three germ layers, as well as teratoma formation and germline transmission in vivo. These data support that converting fibroblasts with Bmil or activation of the sonic hedgehog pathway to an intermediate cell type that expresses Sox2, KIf4, and N-Myc allows iPS generation via the addition of Oct4.

Keywords: reprogramming; transdifferentiation; neural stem cells; induced pluripotent stem cells; Bmi1; Oct4 Cell Research (2011) 21:1305-1315. doi:10.1038/cr.2011.107; published online 28 June 2011

\section{Introduction}

The potential of induced pluripotent stem (iPS) cell technology is enormous, but comprehension of the mo-

Correspondence: Seungkwon You ${ }^{\mathrm{a}}$, Hans R Schöler ${ }^{\mathrm{b}}$, Byung Sun Yoon ${ }^{\mathrm{c}}$

${ }^{a}$ Tel: +82-2-3290-3057; Fax: +82-2-3290-3507

E-mail: bioseung@korea.ac.kr

${ }^{\mathrm{b}}$ Tel: +49-251-70365-300; Fax: +49-251-70365-399

E-mail: office@mpi-muenster.mpg.de

${ }^{\mathrm{c}}$ Tel: +82-2-3290-3493; Fax: +82-2-3290-3507

E-mail: biosun302@korea.ac.kr

Received 9 February 2011; revised 28 March 2011; accepted 4 May 2011; published online 28 June 2011 lecular mechanisms that underlie reprogramming is meager, largely because the procedure is still very inefficient. Recently, a number of groups have demonstrated that the inactivation of p53 markedly increases the efficiency of iPS cell generation [1-6]. Furthermore, by reducing the expression of both $\mathrm{p} 16^{\text {Ink4a }}$ and $\mathrm{p} 19^{\text {Arf }}$ (both of which are encoded by alternative reading frames of the Ink4a/Arf locus, also known as the Cdkn2a locus), iPS cell formation was increased relative to that achieved by reducing the expression of $\mathrm{p} 19^{\text {Arf }}$ alone.

Bmil was first identified as a proto-oncogene that cooperates with c-Myc to promote the formation of B- and T-cell lymphomas [7, 8] by inhibiting c-Myc-induced 
apoptosis through repression of the Ink4a/Arf locus [9, 10]. Bmil is also required for the self-renewal of stem cells and the Ink4a/Arf locus is the main target of Bmi1 stem cell proliferation activity [11-13]. Furthermore, activation of the sonic hedgehog (Shh) signaling pathway induces Bmi1, Sox2, and N-Myc expression, resulting in increased proliferation of neural precursors [14-16]. In this study, we hypothesized that cells with reduced expression of $\mathrm{p} 16^{\mathrm{Ink4a}}$ and $\mathrm{p} 19^{\text {Arf }}$ as well as increased $\mathrm{N}$ Myc, Klf4, and Sox 2 expression [13, 16] mediated by Bmil overexpression could be efficiently converted into iPS cells with either two factors (Oct4 and Sox2: hereafter designated as 2F-Bmil-iPS cells (OSB)) or only one factor (Oct4: hereafter designated as BO-iPS cells). Furthermore, we also asked whether Shh or activation of the Shh signaling pathway by oxysterol or purmorphamine can regulate the downstream target genes of Bmil in the generation of BO-iPS cells. Our study demonstrates that Bmi1 has dual effects on iPS cell generation from fibroblasts by both suppressing p16 ${ }^{\text {Ink4a }}$ and $\mathrm{p} 19^{\text {Arf }}$ and augmenting Sox 2 and N-Myc. By inducing Bmil with some chemicals, we have also shown that only one transcription factor (Oct4) is required to reprogram fibroblasts into pluripotent cells, which have the ability to differentiate into all three germ layer cell types and are capable of germline transmission.

\section{Results}

Bmil replaces the function of Klf4 and $c-M y c$, and increases reprogramming efficiency

Considering that Bmil is essential for the self-renewal of stem cells through repression of the p53 and Rb pathways, we investigated whether Bmil could enhance the reprogramming of fibroblasts into iPS cells. To answer this question, we first determined the expression levels of reprogramming-related genes $\left(\mathrm{p} 16^{\text {Ink4a }}, \mathrm{p} 19^{\text {Arf }}\right.$, Sox2, $\mathrm{N}-\mathrm{Myc}$, p53, and Klf4) in parental mouse embryonic fibroblasts (MEFs) and Bmi1-transduced cells. p16 ${ }^{\text {Ink4a }}$, p19 $9^{\text {Arf }}$, and p53 were significantly repressed in Bmi1transduced cells compared to MEFs; however, consistent with a previous report [16], Sox2, N-Myc, and Klf4 were abundantly expressed (Figure 1A and 1B). Next, we tested whether Bmil could replace Oct4, Sox2, Klf4, or C-Myc. The four transcription factors (Oct4, Sox2, Klf4, and C-Myc; OSKM) or a combination of three of the transcription factors with Bmil (OSKB, OSBM, OBKM, and BSKM) were introduced into MEFs. Bmi1 was able to replace Sox2, Klf4, or C-Myc in inducing Nanog-positive colonies that resemble embryonic stem (ES) cells (Figure 1C and Supplementary information, Figure S1). However, we found that in the absence of
Oct4, Nanog positive colonies were not formed (data not shown), indicating that Bmil is not able to replace Oct4 for reprogramming MEFs. We also tested whether Bmi1 was able to replace both Klf4 and C-Myc (Figure 1A). We reprogrammed mouse fibroblasts with Oct4 and Sox2 or with the two factors plus Bmil (2F-Bmi1-iPS (OSB)). Overexpression of Bmil in these two-factor experiments increased the number of Nanog-positive colonies, consistent with a role of Bmil as a replacement for Klf4 and $\mathrm{C}-\mathrm{Myc}$, as well as a limited role in regulating the p53 and $\mathrm{Rb}$ pathways during reprogramming. The OSB colonies were very similar to those comprised of mouse ES cells and expressed pluripotency-associated transcription factors as well as pluripotent cell surface markers (Figure 1D and Supplementary information, Figure S1). Taken together, these data suggest that Bmil-mediated regulation of N-Myc, Klf4, p16 ${ }^{\text {Ink4a }}$, and p19 ${ }^{\text {Arf }}$ activity markedly increases reprogramming efficiency.

\section{Induction of fibroblasts into iPS cells with Bmil plus Oct4}

Methods designed to reduce the number of factors necessary for reprogramming have taken advantage of endogenously expressed reprogramming factors such as Sox2 [17]. For example, studies show that adult mouse neural stem cells (NSCs), which exhibit endogenous Sox 2 expression, can be reprogrammed by Oct 4 alone [17, 18]. However, methods reprogramming somatic cells that lack endogenous Sox 2 expression to pluripotency with Oct4 alone need to be explored. Based on previous results [16], we hypothesized that cells transduced by Bmil could be transdifferentiated into NSC-like cells and then converted into iPS cells by Oct4 (Figure 2A left panel). We first determined whether Bmil could transdifferentiate MEFs into NSC-like cells. Bmiltransduced MEFs, but not empty vector-transduced MEFs, formed colonies exhibiting an NSC-like morphology within 3-7 days in NSC culture (Figure 2A and 2B). Of the 45 colonies generated, four were selected and grown using standard mouse NSC culturing methods. All four selected colonies expressed genes and cell surface markers characteristic of mouse NSCs, including Nestin and Sox2, as well as AP activity (Figure 2C). In addition, Bmil-transduced spheres gave rise to neurons, oligodendrocytes, and astrocytes (Figure 2D). Next, we investigated whether Bmil-transduced NSC-like cells could be reprogrammed into iPS cells by transduction with Oct4 alone. Indeed, we succeeded in generating ESlike colonies within 10-14 days. We refer to these reprogrammed cells as transdifferentiated BO-iPS cells (dBOiPS cells). These cells were generated from MEFs that were first transdifferentiated into NSC-like cells and then 
A

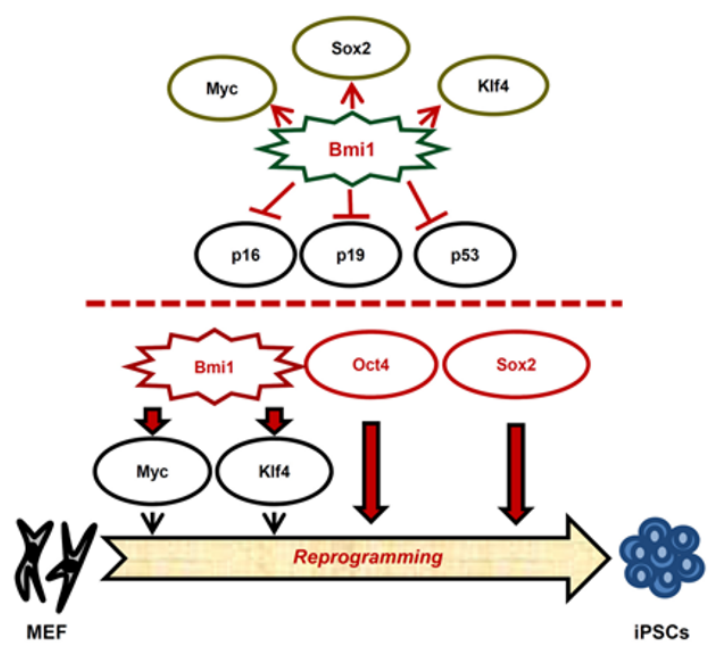

B

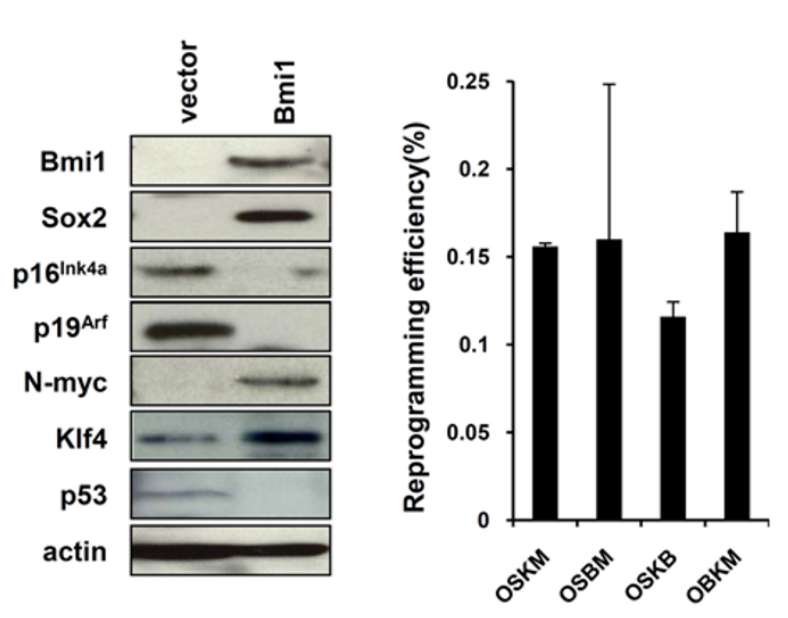

D
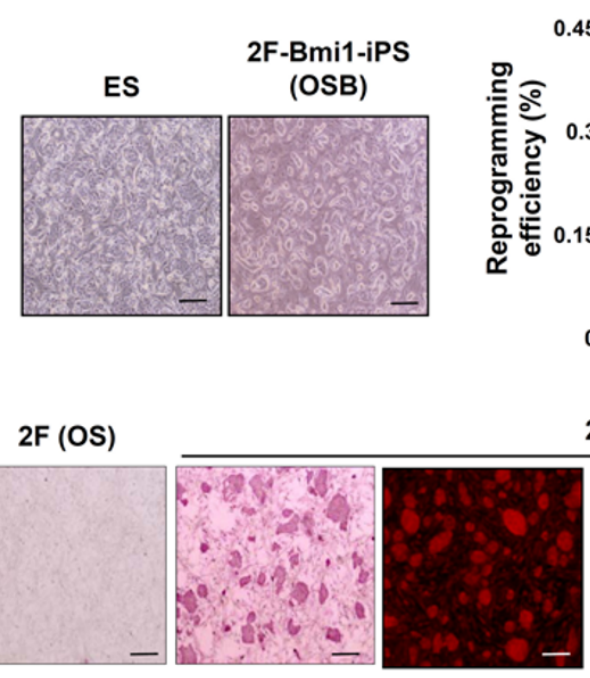

C
$2 \mathrm{~F}(\mathrm{OS})$

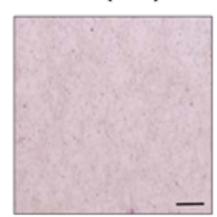

AP
AP

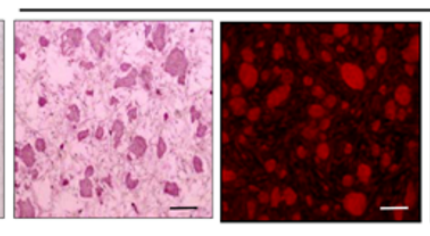

Oct4

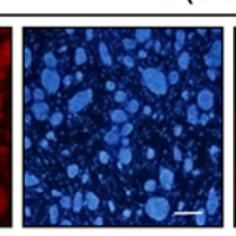

DAPI

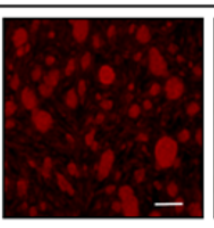

Nanog

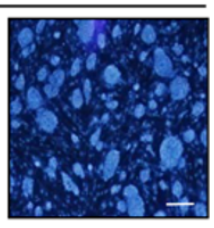

DAPI

Figure 1 Increased generation of iPS cells by overexpression of Bmi1. (A) Hypothesis of Bmi1's function in the course of reprogramming. (B) MEFs were infected with a retrovirus encoding Bmi1 or an empty vector. Three days after infection, protein levels of Bmi1, p16 ${ }^{\text {Ink4a }}$, p19 Arf , and Sox2 were analyzed by western blot. Actin was used as a loading control. (C) Reprogramming efficiencies of factor combinations on iPS cell induction. Bmi1 replaces Sox2, KIf4, and C-Myc in the reprogramming of MEFs. Reprogramming efficiency was quantified by determining the percentage of Nanog-positive colonies. (D) MEFs were infected with retroviruses encoding two factors (Oct4 and Sox2; OS) or with the two factors plus Bmi1 (OSB). Seven days after infection, Nanog-positive colonies formed from cells infected with OSB, but not OS (left and lower panel). Scale bars, 200 $\mu \mathrm{m}$. Protein levels of Oct4, Sox2, c-Myc, KIf4, and p53 were analyzed by western blot. $\alpha$-tubulin was used as a loading control (right panel).

further reprogrammed into iPS cells (Figure 2E). We also determined whether Bmil could replace Sox2, Klf4, and $\mathrm{C}-\mathrm{Myc}$ and, in combination with Oct4, reprogram MEFs into iPS cells (Figure 2A right panel). We introduced Bmil and Oct4 into MEFs and were able to induce the formation of ES-like cells, hereafter designated as BOiPS cells. The estimated reprogramming efficiency was calculated at $0.01 \%$ and $0.17 \%$ for $\mathrm{dBO}-$ and $\mathrm{BO}-\mathrm{iPS}$ cells, respectively. The reprogramming efficiency of dBO-iPS cells was approximately $6 \%$ of that obtained 

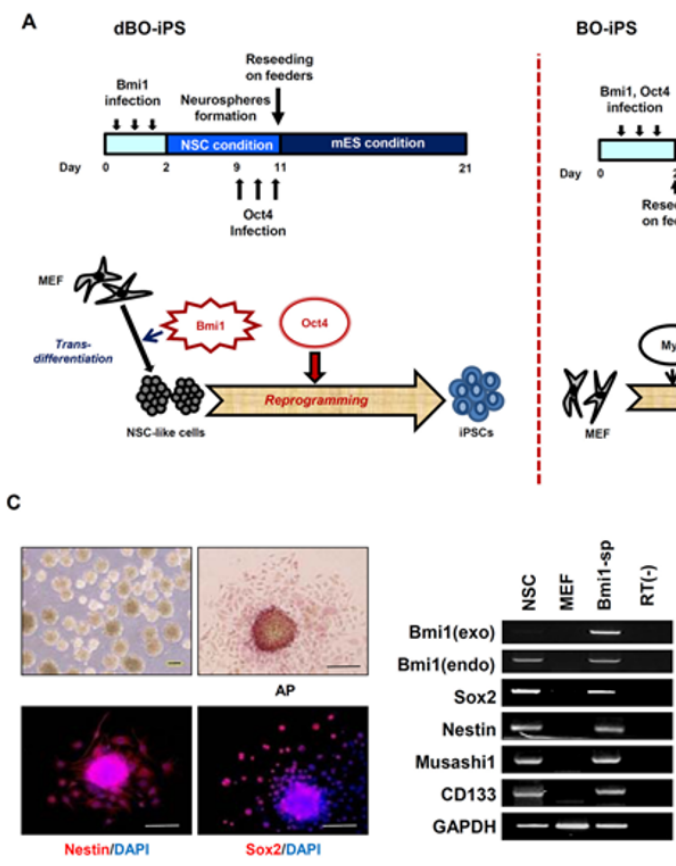

E
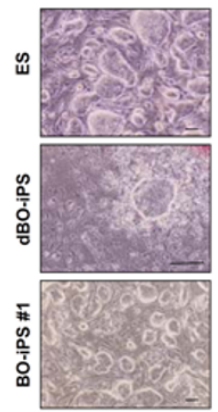

$\mathbf{F}$

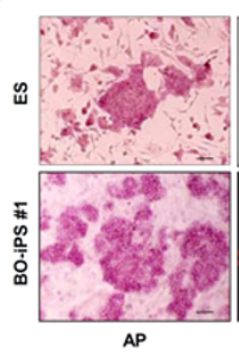

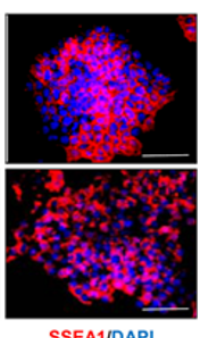

SSEA1/DAPI
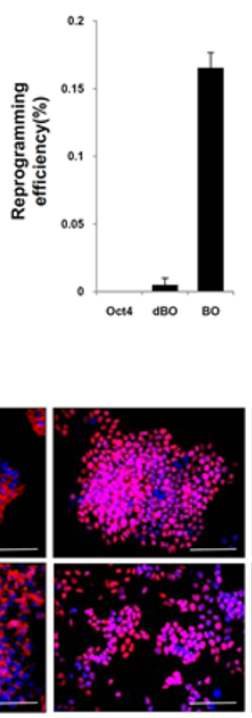

Oct4/DAPI
BO-iPS

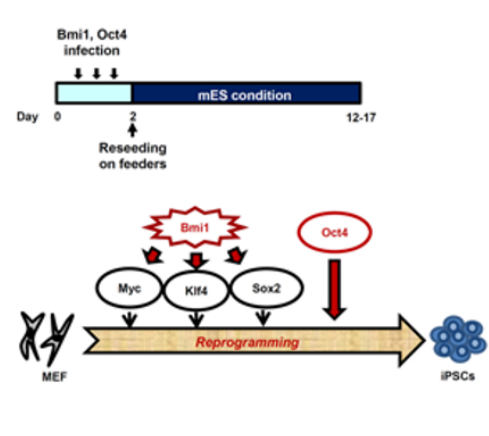

D
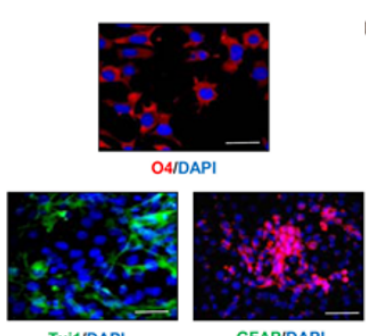

GFAPIDAPI

G

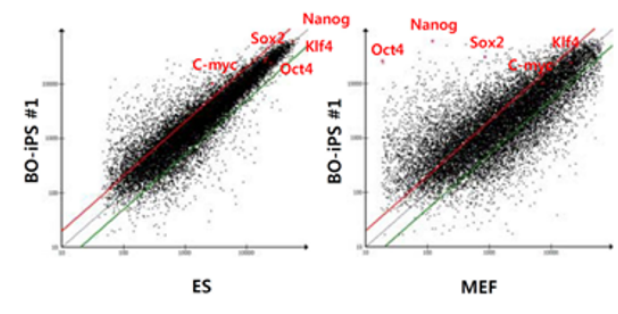

H

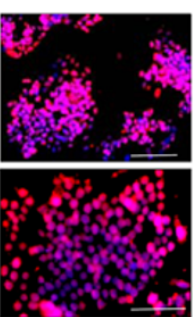

Sox2/DAPI

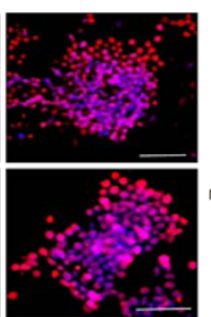

Nanog/DAPI
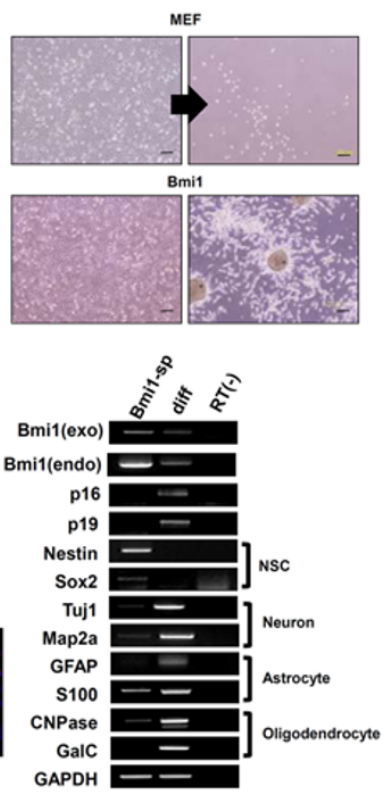

Figure 2 Characterization of iPS cells generated from MEFs by retroviral transduction with Oct4 and Bmi1. (A) Hypothesis of Bmi1's function in the course of reprogramming MEFs into dBO- (left) and BO-iPS cell (right). (B) Phase contrast images of vehicle- (upper) and Bmi1-transduced (lower) MEFs cultured in proliferation medium (left) and in NSC medium (right). Bmi1-transduced MEFs cultured in NSC medium for 3-7 days rapidly changed morphology, resulting in bipolar and expanded NSC-like cells (right). Scale bars, $200 \mu \mathrm{m}$. (C) Characterization of NSC-like cells was conducted by AP staining and immunocytochemistry for Nestin and Sox2, as well as RT-PCR analysis of NSC marker genes (right). Scale bars, $200 \mu \mathrm{m}$. (D) NSClike cells exhibit multipotency and thus can give rise to cells expressing neuronal and glial markers for oligodendrocytes (O4, CNPase, and GalC), neurons (Tuj1 and Map2a), and astrocytes (GFAP and S100). Scale bars, $200 \mu \mathrm{m}$. (E) Phase contrast images showing mES cells (upper left) and BO-iPS cells (clone 1; lower left) on feeder cells. The reprogramming efficiency of $\mathrm{dBO}$ - and BO-iPS cells was quantified by determining the percentage of Nanog-positive colonies (right). (F) Characterization of BO-iPS-1 cells. AP staining, as well as SSEA1, Oct4, Sox2, and Nanog immunoreactivities, were measured in mES cells (upper panels) and BO-iPS cells (lower panels). Scale bars, $200 \mu \mathrm{m}$. (G) Scatter plots of the global gene expression comparing BO-iPS cells with MEFs (right) and BO-iPS cells with mES cells (left). Red and green lines indicate 2-fold changes in gene expression levels. The pluripotency genes Oct4, Sox2, Nanog, c-Myc, and Klf4 are shown in red. (H) Bisulfite genomic sequencing of Oct4 and Nanog promoter regions in mES cells, MEFs, and BO-iPS clones (1, 2, and 3). Open and filled circles indicate unmethylated and methylated $\mathrm{CpG}$ dinucleotides, respectively. 
by BO-iPS cells. The reason for the lower reprogramming efficiency of dBO-iPS than BO-iPS cells is not clear; however, it may be due to the poor transduction efficiency of Oct4 into the NSC-like spheres. To further characterize the BO-iPS cells, six colonies that were morphologically indistinguishable from mouse ES (mES) cell colonies were selected and grown under standard conditions (Figure 2E). BO-iPS cells expressed marker genes at levels typical of mES cells (Figure 2F and Supplementary information, Figures S2A, B, and S3A, B). Furthermore, scatter plots of the global gene expression profiles showed that the BO-iPS cell profile was similar to mES cells and was different from MEFs (Figure 2G). To further compare BO-iPS cells with mES cells, the methylation state of $\mathrm{CpG}$ dinucleotides in the Oct4 and Nanog promoter regions was analyzed. Bisulfite genomic sequencing analyses showed that the Oct4 and Nanog promoter regions were demethylated in BO-iPS cells relative to the parental fibroblasts and showed a similar pattern to that of mES cells (Figure 2H). Chromatin immunoprecipitation (ChIP) analyses showed that the Oct4, Sox2, and Nanog promoters had increased acetylation of histone $\mathrm{H} 3(\mathrm{AcH} 3)$ and decreased dimethylation of lysine 9 of histone H3 (diMeK9H3) (Supplementary information, Figure S2C), consistent with the epigenetic remodeling that occurs during reprogramming. Oct4 mRNA was transcribed from the endogenous Oct4 locus, which can be distinguished from the virally expressed human Oct4. Genomic integration of the Bmil and Oct4 transgenes was confirmed (Supplementary information, Figure S3C), and the expression of both transgenes was efficiently silenced in all iPS cell lines examined (Supplementary information, Figure S3D) [19].

We next investigated the differentiation potential of BO-iPS cells in vitro with the embryoid body (EB) assay. EBs derived from BO-iPS cells expressed markers of the three germ layers, including the endoderm marker GATA4, the mesoderm markers smooth muscle actin and Brachyury, and the ectoderm marker Nestin (Figure $3 \mathrm{~A})$. To investigate the differentiation potential of BOiPS cells in vivo, we analyzed teratomas that formed after subcutaneous injection of BO-iPS cells into nude mice. These teratomas contained derivatives of the three embryonic germ layers, including neural epithelium, muscle, cartilage, and various glandular structures (Figure 3B and Supplementary information, Figure S3E). To assess their developmental potential, we tested whether BOiPS cells could generate chimeric mice after injection into blastocysts. According to coat color, BO-iPS clones generated chimeric mice with germline transmission (Figure 3C). Taken together, these results demonstrate that Bmil can either transdifferentiate MEFs into NSC- like cells and/or replace Sox2, Klf4, and C-Myc during reprogramming of MEFs into iPS cells in the presence of Oct4. In addition, our results suggest that chemically induced activation of Bmil may be a useful strategy for enhancing reprogramming efficiency without genetic manipulation of the potential oncogenic Bmil.

Shh induces Bmil and stimulates the generation of iPS cells from fibroblasts by transduction with Oct4 alone

Shh, the most prominent member of the Hedgehog family, plays an essential role during development. The Shh signaling pathway involves the activation of Gli transcription factors, which regulate the transcription of target genes including Gli1 and Ptch1. Furthermore, Bmi1, Sox2, and N-Myc expression was upregulated in response to Shh treatment and parallels the expression of Gli1, suppressor of fused (Sufu), and cyclin D2, which is indicative of the activation of the Shh pathway and induction of proliferation [14-16, 20, 21]. Moreover, overexpression of Gli1 induces Bmil expression, suggesting that Bmil is a downstream target in the Shh pathway [15].

Therefore, we tested whether Shh could replace Bmil in the generation of dBO- and BO-iPS cells. Bmi1, Sox2, $\mathrm{N}-\mathrm{Myc}$, Klf4, and Gli1 mRNAs were upregulated (in contrast to $\mathrm{p} 16^{\text {Ink4a }}$ and $\mathrm{p} 19^{\text {Arf }}$ mRNAs, which were suppressed) in response to Shh treatment as early as $72 \mathrm{~h}$ after incubation, indicating the activation of the Shh pathway (Figure 4A and 4B). Moreover, cells developed into colonies exhibiting an NSC-like morphology within 3-7 days of Shh treatment in standard NSC culture conditions (Figure 4C). These NSC-like cells expressed genes and cell surface markers characteristic of mouse NSCs, including Sox2, Nestin, and SSEA1, as well as AP activity (Figure 4D). Shh-treated NSC-like cells were then transduced with Oct4 to reprogram them into iPS cells (1F combination of Shh and Oct4, hereafter designated as ShO-iPS cells). ShO-iPS colonies obtained within 14 days in culture were further analyzed by the same tests described above for BO-iPS cells to confirm reprogramming to pluripotency (Figure 4E-F and Supplementary information, Figure S4). Taken together, these results demonstrate that Shh can induce Bmil, and together with Oct4, can reprogram MEFs into iPS cells that are very similar to mES cells (Supplementary information, Figure S4I).

Recently, it was demonstrated that specific oxysterol and purmorphamine not only stimulate the Shh pathway but also activate Shh target gene transcription through the protein Smo $[22,23]$. Similar to ShO-iPS cells, treatment of MEFs with either oxysterol or purmorphamine activated the Shh pathway, reprogramming MEFs into NSC-like cells that exhibited gene expression profiles 
A

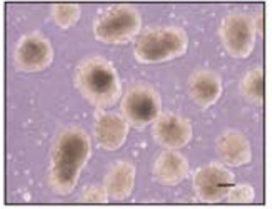

EB

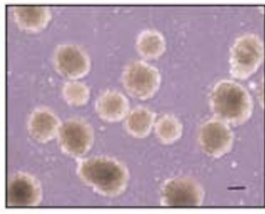

BO-iPS-EB \#1

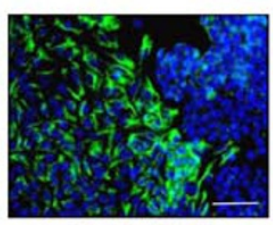

Nestin/DAPI

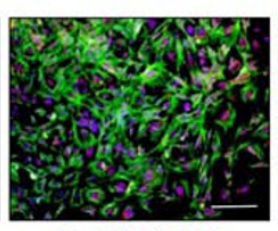

BryISMAIDAPI
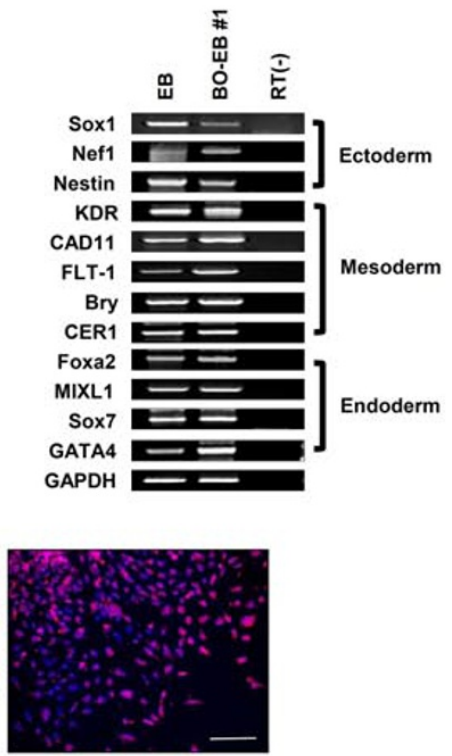

GATA4/ DAPI

B

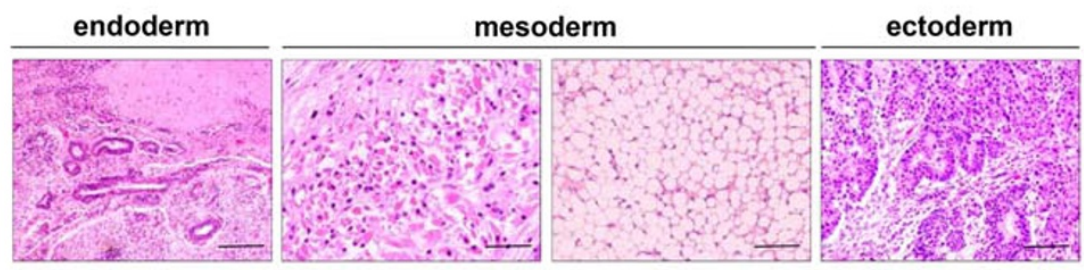

C
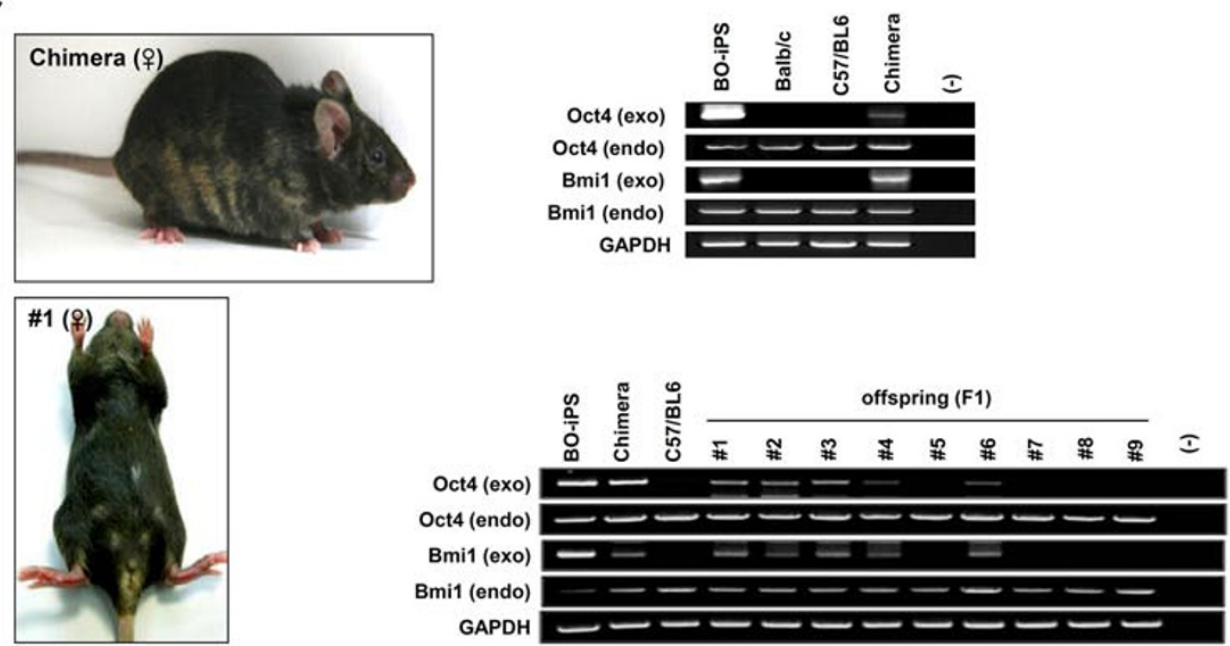

Figure 3 In vitro and in vivo differentiation of BO-iPS cells. (A) In vitro differentiation of BO-iPS cells. Micrographs show EBs generated from BO-iPS-1 clones and their in vitro differentiation into ectodermal, mesodermal, and endodermal cell types, as revealed by immunoreactivity to typical markers Nestin, Brachyury, SMA, and GATA4, respectively. Nuclei were counterstained with DAPI (blue). Scale bars, $200 \mu \mathrm{m}$. RT-PCR analysis shows that cDNAs from EBs exhibit expression of representative lineage markers in differentiating cells. (B) The in vivo developmental potential of BO-iPS cells. Teratomas generated by BO-iPS cells differentiated into neural rosettes (ectoderm), muscle and fat (mesoderm), and epithelium (endoderm). Hematoxylin and eosin-stained sections of teratomas derived from BO-iPS cells in a nude mouse after 8-10 weeks are shown. Scale bars, $200 \mu \mathrm{m}$. (C) Chimeric mouse (upper panels) and germline contribution of BO-iPS cells in adult chimera gonads (lower panels). Established iPS cells give rise to live chimeras (upper left) after an injection of BO-iPS cells (Balb/c genetic background) into a C57BL6 blastocyst and contribute to the germline (lower left). PCR was performed on genomic DNA to detect exogenous and endogenous transgene integration in chimeric (upper right) and germline transmission mice (lower right) produced with BO-iPS cells. 
A
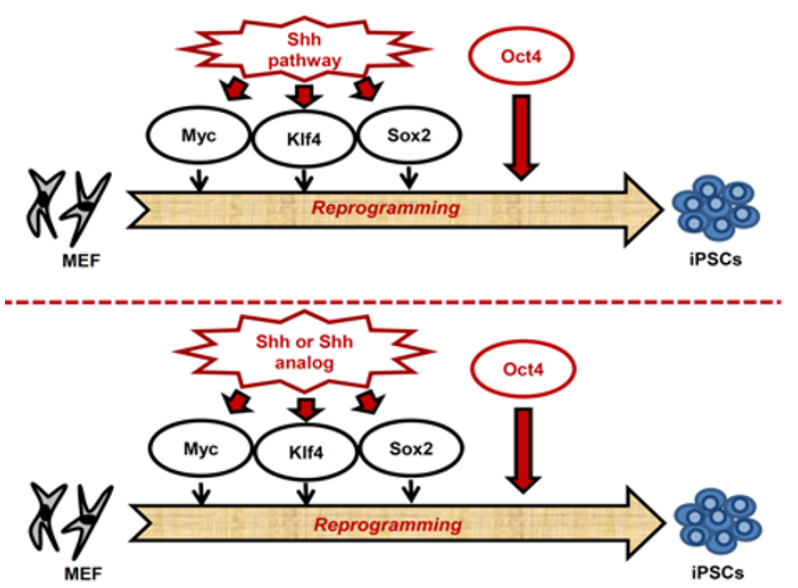

B
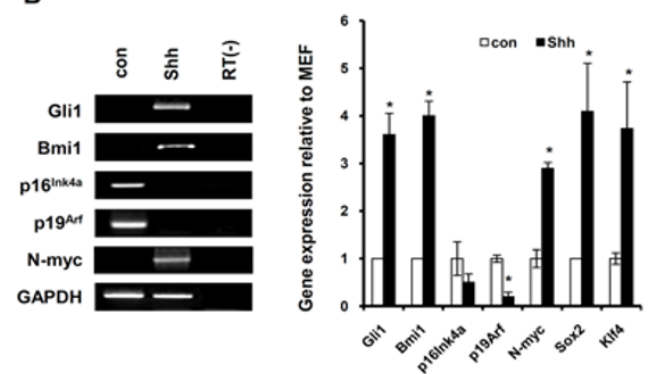

C

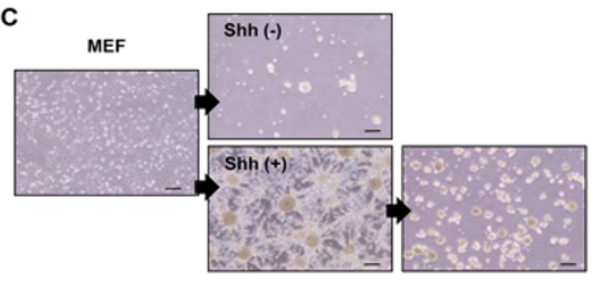

D

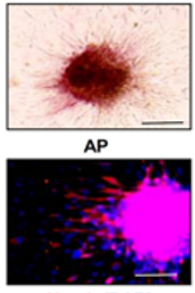

Nestin/DAPI

E

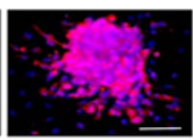

SSEA1/DAPI

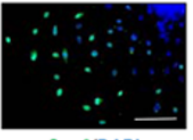

Sox2IDAPI
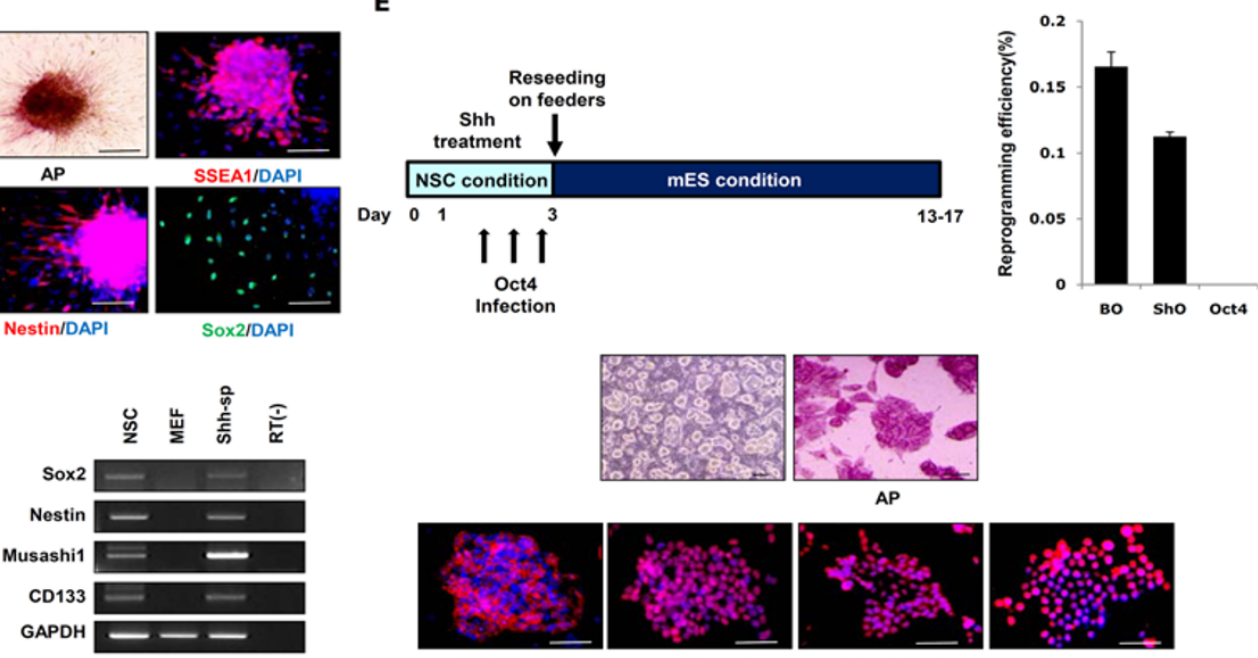

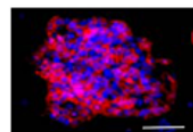

SSEA1/DAPI

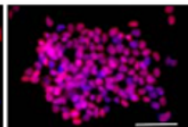

Oct4/DAPI

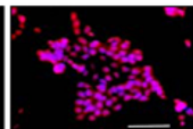

Sox2/DAPI

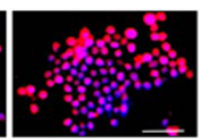

Nanog/DAPI

F

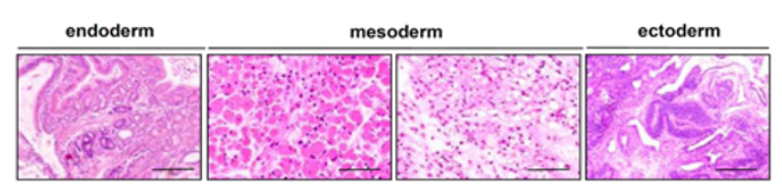

Figure 4 Generation and characterization of 1F ShO-iPS cells. (A) Hypothesis of induction of Bmi1 in the course of reprogramming. (B) Induction of sonic hedgehog target genes by Shh treatment. RT-PCR and qPCR of mRNAs from MEFs treated with vehicle (con) or Shh were analyzed for the induction of Shh target genes (Gli1, Bmi1, Sox2, and N-Myc, as well as $\mathrm{p} 16^{\mathrm{Ink} 4 \mathrm{a}}, \mathrm{p} 19^{\mathrm{Arf}}$, and KIf4). Data are from a representative experiment (left) and are shown as the means and SD's of relative values compared to control MEFs $(n=3)$. ${ }^{*} P<0.05$ compared to MEFs. (C) Phase contrast images of vehicle- $($ shh $(-))$ and Shh-treated $(\mathrm{shh}(+))$ MEFs cultured in proliferation medium (left) or NSC medium (right). Shh-treated MEFs cultured in NSC medium for 7 days rapidly changed morphology, resulting in bipolar (right) and expanded neurosphere-like cells (right). Scale bars, $200 \mu \mathrm{m}$. (D) Characterization of neurosphere-like morphology from Shh-treated MEFs was conducted by AP staining, immunocytochemistry, and RT-PCR for Nestin, Sox2, SSEA1, Musashi1, and CD133. Scale bars, $200 \mu \mathrm{m}$. (E) Timeline for ShO-iPS cell induction using Shh treatment with retroviral transduction of Oct4 (upper and left, panel). Phase contrast images showing the ESC-like morphology of ShO-iPS cells on feeder cells and the characterization of ShO-iPS cells. AP staining, as well as SSEA1, Oct4, Sox2, and Nanog immunoreactivity, was detected in ShO-iPS cells (lower panel). The reprogramming efficiency of BO- and ShO-iPS cells in reprogramming MEFs. Reprogramming efficiency was quantified by determining the percentage of Nanog-positive colonies. Scale bars, $200 \mu \mathrm{m}$ (upper and right panel). (F) The in vivo developmental potential of ShO-iPS cells. Teratomas of ShO-iPS cells differentiated into epithelium (endoderm; left), muscle and fat (mesoderm; middle), and neural rosettes (ectoderm; right). Hematoxylin and eosin-stained sections of teratomas derived from ShO-iPS cells in a nude mouse host after 8 weeks are shown. Scale bars, $200 \mu \mathrm{m}$. 
characteristic of NSCs (Supplementary information, Figure S5A-S5C). Furthermore, the treatment of MEFs with oxysterol and/or purmorphamine enhanced the reprogramming of MEFs to pluripotency by the forced expression of Oct4 (1F combinations of oxysterol and/or purmorphamine and Oct4, hereafter designated as OxyOiPS, PO-iPS, or POxyO-iPS cells) (Supplementary information, Figure S5D). Again, the tests described above were successfully conducted with PO-iPS and OxyO-iPS cells (Supplementary information, Figures S5E-M and
S6A-H). Furthermore, PO-iPS cells were germline competent, as demonstrated by the generation of albino offspring from crossing chimeric mice with wild-type mice (Supplementary information, Figure S6I). These results demonstrate that MEFs can be reprogrammed to pluripotency by Oct4 alone when the Shh pathway is activated.

Given that Bmil is an important regulator of reprogramming-related genes (Figure 1A and 1B) [13], the transdifferentiation of MEFs into NSC-like cells, and the generation of iPS cells with Oct4, we studied whether
A

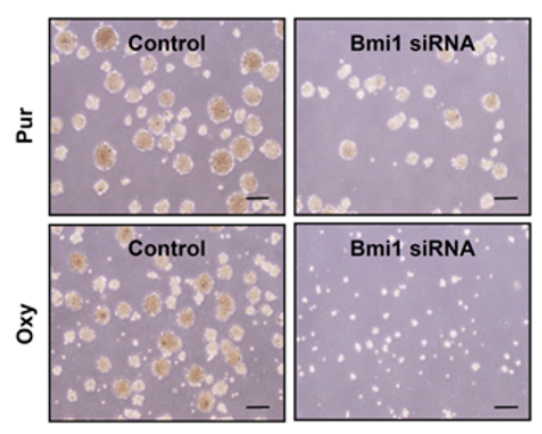

B

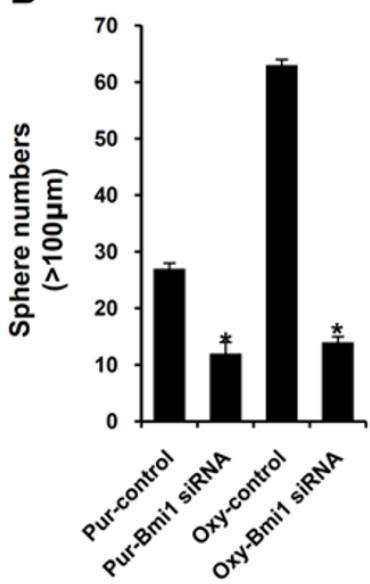

E

D
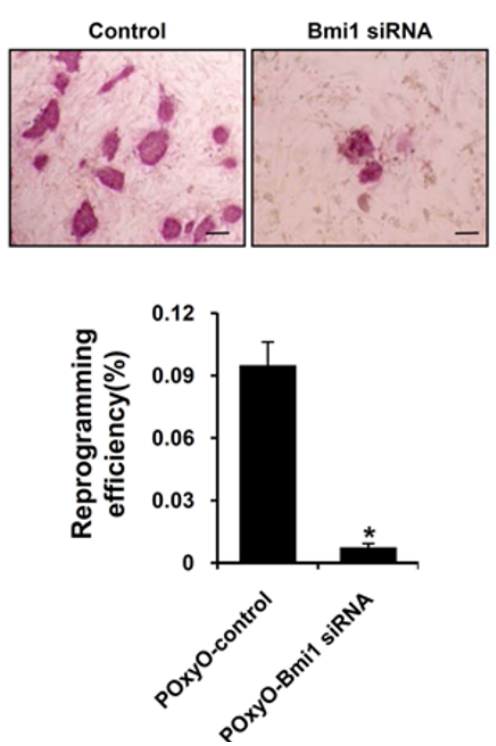

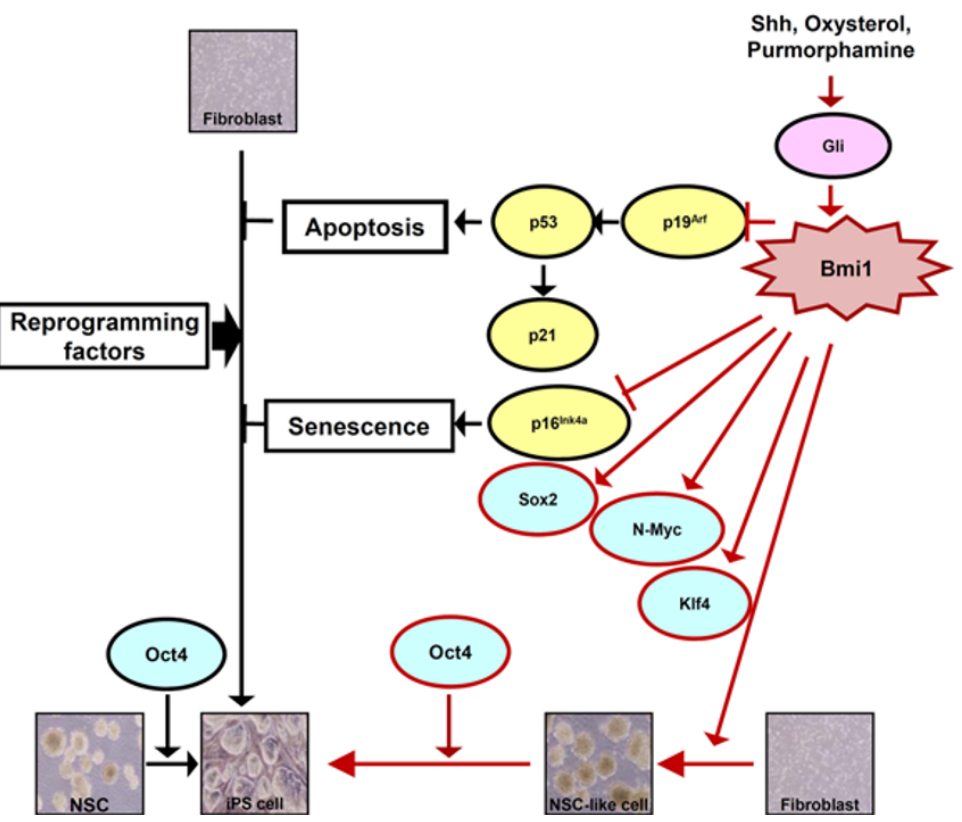

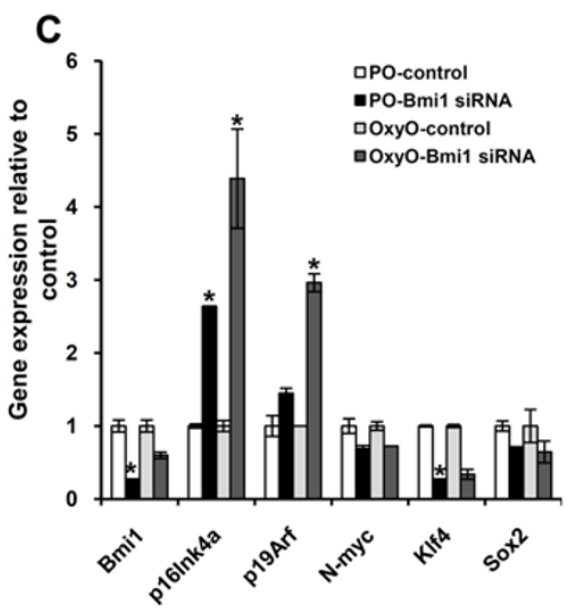

Shh, Oxysterol,

Figure 5 Decreased generation of iPS cells by the knockdown of Bmi1. (A, B) Inhibition of Bmi1 expression prevents neurosphere formation. Scale bars, $200 \mu \mathrm{m}$. (C) qPCR analysis of Bmi1 target genes in vehicle and Bmi1 siRNA-transfected purmorphamine- or oxysterol-treated MEFs. ${ }^{*} P<0.05$ compared to control. (D) The reprogramming efficiency of vehicle and Bmi1 siRNA-transfected POxyO-iPS cells in reprogramming MEFs. Reprogramming efficiency was quantified by determining the percentage of Nanog-positive colonies. (E) Model summarizing the presented data. Bmi1 and Bmi1 inducers (Shh, purmorphamine, and oxysterol) enhance Oct4-induced reprogramming of fibroblasts by downregulating $\mathrm{p} 16^{\text {Ink4a }}$ and $\mathrm{p} 19^{\text {Aff }}$ expression and upregulating Sox2, N-Myc, and Klf4 expression. 
knocking down Bmil expression would blunt neural sphere formation. Transdifferentiation was performed in the presence of oxysterol and/or purmorphamine to induce the transdifferentiation of MEFs into NSC-like cells. The formation of neurospheres and reprogramming-related gene expression were significantly altered by the knock down of Bmil (Figure 5A-5C). Moreover, when cells treated with both oxysterol and purmorphamine were transduced with Oct4 in the presence of Bmil siRNA, the formation of Nanog-positive colonies was significantly decreased compared to control (Figure 5D).

We next tested the combination of oxysterol and Oct4 on the reprogramming of tail-tip fibroblasts (TTFs) from 10 -week-old male mice (hereafter designated as OxyOiPS-TTF cells) and established two independent iPS cell lines. These cells were very similar to ES cells with an identical differentiation potential (Supplementary information, Figure S7). Growth rates and hierarchial clustering analysis of the global gene expression profiles showed that $1 \mathrm{~F}$ (Oct4) and 2F (Oct4 and Bmi1) iPS cells clustered close to $\mathrm{mES}$ cells and were distinct from the parental MEFs and Bmil-transduced neurospheres (Supplementary information, Tables S1-S2 and Supplementary information, Figure S8). The chromosomal stability of $1 \mathrm{~F}$ and $2 \mathrm{~F}$ iPS cells was confirmed by metaphase spread (Supplementary information, Figure S9). Taken together, these results show that Bmil promotes iPS cell generation from fibroblasts; Bmil likely has dual effects on iPS cell generation from fibroblasts, by both suppressing $\mathrm{p} 16^{\text {Ink4a }}$ and $\mathrm{p} 19^{\text {Arf }}$ and augmenting Sox 2 and N-Myc (Figure 5E).

\section{Discussion}

In summary, our data support two main conclusions. First, Bmi1 can replace Sox2, Klf4, and C-Myc during reprogramming and enhances the reprogramming efficiency of mouse fibroblasts infected with Oct4 and Sox2. This suggests that not only p16 $6^{\text {Ink4a }}$ and $\mathrm{p} 19^{\text {Arf }}[1-4,6]$, but also Sox 2 and C-Myc [17-19, 24] are rate-limiting determinants in the reprogramming process. The second conclusion relates to the number of factors applied to somatic cells for iPS cell generation. We demonstrated previously that mouse and human NSCs, which express endogenous Sox2, can be reprogrammed with Oct4 alone $[17,18]$. Here, we expand upon these results by showing that Oct4, together with Bmil or activators of the Shh signaling pathway (Shh, oxysterol, and purmorphamine in this study), is sufficient for the generation of iPS cells from mouse embryonic and adult fibroblasts. This is especially crucial because adult fibroblasts do not express Sox2. This study, together with others is an important step forward in defining the critical determinants for the generation of iPS cells from differentiated fibroblasts [25-27]. Future studies will determine if we can combine our direct reprogramming procedure with small molecule compounds to activate endogenous expression of Oct4, knock down Oct4-specific suppressor(s), or achieve reprogramming with Oct4 recombinant protein alone.

\section{Materials and Methods}

\section{Generation of $4 F$ and $2 F$ iPS cells}

pBabe-based retroviral vectors encoding Oct4, Sox2, Klf4, or C-Myc (from Gou Young Koh), and Bmil (from Goberdhan P. Dimri) were transfected into PT67 amphotropic packaging cells (Clontech) using Turbofect (Fermentas) according to the manufacturer's protocol. The cells were subjected to drug selection with $3 \mu \mathrm{g} / \mathrm{ml}$ puromycin (Bmi1, Klf4 and C-Myc) for 4 days or 1 $000 \mathrm{ng} / \mathrm{ml} \mathrm{G} 418$ (Sox 2 and Oct4) for 14 days. The viruses were collected after $24 \mathrm{~h}$ and filtered through a $0.45-\mu \mathrm{m}$ filter before transduction. Induction of iPS cells was performed as described previously [24] with some modification. In brief, MEF cells $(2 \times$ $10^{5}$ ) were seeded in one well of a six-well plate and $2 \mathrm{ml}$ of each retroviral supernatant (Bmil and Oct4) was added to the cells in the presence of $6 \mu \mathrm{g} / \mathrm{ml}$ of polybrene (Sigma). Two days after the initial transduction, cells were subcultured on mitomycin C-treated CF1 mouse feeder layers and maintained in mES cell medium consisting of DMEM supplemented with 15\% FBS (Hyclone), $\beta$-mercaptoethanol, $1 \%$ penicillin-streptomycin, and 1000 units/ $\mathrm{ml}$ leukemia inhibitory factor (LIF; Millipore). ES and iPS cells were passaged every $3-5$ days using $0.05 \%$ trypsin-EDTA and seeded at $2 \times 10^{5}$ cells/well in a six-well plate. The induction of $\mathrm{dBO}$-iPS cells was performed in two steps. Generation of NSClike cells was achieved with MEFs infected with retroviruses containing Bmi1, as described previously [16], and these NSC-like cells were then further infected with retroviruses containing Oct4, as above. Two days after Oct4-transduction, cells were grown in mES-culture conditions as described above. For the quantification of transduction efficiency, we introduced GFP to MEF as above and more than $70 \%$ of cells expressed GFP. To quantify efficiency of iPS generation, total number of Nanog-positive colonies were counted and calculated as percentage of infected cell numbers, which was calculated as GFP+ cells [28].

\section{Generation of $1 F$ iPS cells}

To induce ShO-, PO-, OxyO-iPS, and OxyO-iPS-TTF cells, cells were cultured in NSC medium [16] with either Shh (500 ng/ $\mathrm{ml}$; R\&D systems), purmorphamine $(0.5,1 \mu \mathrm{M}$; Calbiochem), or 25-hydroxycholesterol (oxysterol; $0.1,0.5 \mu \mathrm{M}$; Sigma) for 1 day. Next, retroviral supernatants (Oct4) in NSC medium containing $6 \mu \mathrm{g} / \mathrm{ml}$ polybrene were added to the cells with Shh, purmorphamine, or oxysterol. A third round of transduction was performed and the cells were incubated for 2 days. Two days after the initial transduction, cells were transferred and grown on mitomycin C-treated MEF layers in mES cell medium. For siRNA experiments, cells were transfected with lipofectamine RNAiMAX (Invitrogen). Bmil siRNAs and control siRNAs were purchased from Bioneer. 


\section{Microarray analysis}

Total RNA from MEF, mES, and iPS cells was labeled with Cy3. Labeled RNA was hybridized to the Agilent mouse wholegenome array (G4122F) according to the manufacturer's instructions. Arrays were scanned with the G2565BA Microarray Scanner System (Agilent Technologies). All data normalization and gene selection was performed using GeneSpring GX 7.3 (Agilent Technologies) [24].

\section{Bisulfite sequencing analysis}

DNA from MEF, mES, and iPS cells was isolated using the Genomic DNA Purification Kit (Promega). DNA was prepared for bisulfite sequencing with the EpiTect Bisulfite Kit (QIAGEN). Treated DNA was used to amplify the sequences of interest. The primers used for promoter fragment amplification are listed in Supplementary information, Table S3. The resulting fragments were cloned using the pGEM-T Easy Vector (Promega) for sequencing and sequenced with T7 forward and SP6 reverse primers.

\section{ChIP assay}

The ChIP assay was performed on MEFs, ES cells, and iPS cells using the EZ ChIP Kit (Millipore) according to the manufacturer's instructions. Anti-acetyl H3 and anti-dimethyl K9 H3 antibodies were used in this experiment. PCR primers used for realtime PCR are listed in Supplementary information, Table S3.

\section{Differentiation of iPS cells}

iPS cells were examined by the in-vitro differentiation of EBs. iPS cells were trypsinized and single cells were cultured in suspension with EB medium without LIF for 7 days, and EBs were then replated onto $0.1 \%$ gelatin-coated plates. Spontaneous differentiation was examined by immunostaining and RT-PCR for representative lineage-specific markers with the indicated antibodies and primers at various timepoints (5-7 days).

\section{Teratoma formation}

iPS cells $\left(1 \times 10^{6} /\right.$ mouse $)$ were injected under the kidney capsule or subcutaneously into the dorsal flank of nude mice. Eight to ten weeks later, the mice developed teratomas, which were removed, immediately rinsed with PBS, fixed in $10 \%$ formalin, and embedded in paraffin. Tissue sections 5-6 $\mu \mathrm{m}$ thick were cut and processed for hematoxylin-eosin staining.

\section{Chimera formation and germline transmission}

Four- to five-week-old female mice (C57BL/6) were induced to superovulate by intraperitoneal injection of 7.5 IU PMSG followed $48 \mathrm{~h}$ later by intraperitoneal injection of $7.5 \mathrm{IU}$ hCG and mated with a stud male mouse $(\mathrm{C} 57 \mathrm{BL} / 6)$. Blastocysts were collected 3.5 days after vaginal plug check and flushing in $\mathrm{H}-\mathrm{CZB}$ medium. Approximately 10 iPS cells then were expelled from the injection pipette against the inner cell mass of the blastocyst. Injected blastocysts were transferred into the uterine horn of 2.5 days post coitum (dpc) pseudopregnant CD1 female mice that had been mated with vasectomized male mice. Chimerism was ascertained by the contribution rate of albino coat color (from iPS cells) in black host pups. High-contribution chimeras were mated with C57BL/6 mice to test for germline transmission.

\section{Statistical analysis}

Data were analyzed by analysis of variants using the general linear model procedures of the Statistical Analysis System (SAS, 9.13 PACKAGE). Data were expressed as the means \pm SD. $P<0.05$ was considered significant.

\section{Accession codes}

Gene Expression Omnibus (GEO): GSE24208

Further details and other methods can be found in the supplementary information, Data S1.

\section{Acknowledgments}

We are grateful to Drs Goberdhan P Dimri (NorthShore University HealthSystem Research Institute, USA)and Gou Young Koh (Korea Advanced Institute of Science and Technology, Republic of Korea) for kindly providing the pBabe-Bmi1, pMX-Oct4, pMXSox2, pMX-K1f4, and pMX-C-Myc constructs. We thank Jihyun Kim, Jihye Hwang, and Suhyun Kwon for technical support. This research was supported by a grant (SC-5150) from the Stem Cell Research Center of the 21st Century Frontier Research Program funded by the Ministry of Education, Science and Technology, Republic of Korea, a grant (09172KFDA653) from the Korea Food and Drug Administration, and a grant (2010-0020347) from National Research Foundation (NRF) funded by the Korea government (MEST). Work in Germany was supported by the Max Planck Society and the Federal Ministry of Education and Research (BMBF) on Cell-Based Regenerative Medicine (Grant 01GN0539).

\section{References}

1 Hong H, Takahashi K, Ichisaka T, et al. Suppression of induced pluripotent stem cell generation by the p53-p21 pathway. Nature 2009; 460:1132-1135.

2 Kawamura T, Suzuki J, Wang YV, et al. Linking the p53 tumour suppressor pathway to somatic cell reprogramming. Nature 2009; 460:1140-1144.

3 Li H, Collado M, Villasante A, et al. The Ink4/Arf locus is a barrier for iPS cell reprogramming. Nature 2009; 460:11361139.

4 Marion RM, Strati K, Li H, et al. A p53-mediated DNA damage response limits reprogramming to ensure iPS cell genomic integrity. Nature 2009; 460:1149-1153.

5 Pei D. Regulation of pluripotency and reprogramming by transcription factors. J Biol Chem 2009; 284:3365-3369.

6 Utikal J, Polo JM, Stadtfeld M, et al. Immortalization eliminates a roadblock during cellular reprogramming into iPS cells. Nature 2009; 460:1145-1148.

7 Haupt Y, Alexander WS, Barri G, Klinken SP, Adams JM. Novel zinc finger gene implicated as myc collaborator by retrovirally accelerated lymphomagenesis in E mu-myc transgenic mice. Cell 1991; 65:753-763.

8 van Lohuizen M, Verbeek S, Scheijen B, Wientjens E, van der Gulden H, Berns A. Identification of cooperating oncogenes in E mu-myc transgenic mice by provirus tagging. Cell 1991; 65:737-752.

9 Jacobs JJ, Kieboom K, Marino S, DePinho RA, van Lohuizen $\mathrm{M}$. The oncogene and Polycomb-group gene bmi-1 regulates cell proliferation and senescence through the ink4a locus. $\mathrm{Na}$ - 
ture 1999; 397:164-168.

10 Jacobs JJ, Scheijen B, Voncken JW, Kieboom K, Berns A, van Lohuizen M. Bmi-1 collaborates with c-Myc in tumorigenesis by inhibiting c-Myc-induced apoptosis via INK4a/ARF. Genes Dev 1999; 13:2678-2690.

11 Molofsky AV, Pardal R, Iwashita T, Park IK, Clarke MF, Morrison SJ. Bmi-1 dependence distinguishes neural stem cell self-renewal from progenitor proliferation. Nature 2003; 425:962-967.

12 Park IK, Qian D, Kiel M, et al. Bmi-1 is required for maintenance of adult self-renewing haematopoietic stem cells. Nature 2003; 423:302-305.

13 Valk-Lingbeek ME, Bruggeman SW, van Lohuizen M. Stem cells and cancer; the polycomb connection. Cell 2004; 118:409-418.

14 Hatton BA, Knoepfler PS, Kenney AM, et al. N-myc is an essential downstream effector of Shh signaling during both normal and neoplastic cerebellar growth. Cancer Res 2006; 66:8655-8661.

15 Leung C, Lingbeek M, Shakhova O, et al. Bmil is essential for cerebellar development and is overexpressed in human medulloblastomas. Nature 2004; 428:337-341.

16 Moon JH, Yoon BS, Kim B, et al. Induction of neural stem cell-like cells (NSCLCs) from mouse astrocytes by Bmil. Biochem Biophys Res Commun 2008; 371:267-272.

17 Kim JB, Sebastiano V, Wu G, et al. Oct4-induced pluripotency in adult neural stem cells. Cell 2009; 136:411-419.

18 Kim JB, Greber B, Arauzo-Bravo MJ, et al. Direct reprogramming of human neural stem cells by OCT4. Nature 2009; 461:649-643.

19 Huangfu D, Osafune K, Maehr R, et al. Induction of pluri- potent stem cells from primary human fibroblasts with only Oct4 and Sox2. Nat Biotechnol 2008; 26:1269-1275.

20 Dahmane N, Ruiz i Altaba A. Sonic hedgehog regulates the growth and patterning of the cerebellum. Development 1999; 126:3089-3100.

21 Wechsler-Reya RJ, Scott MP. Control of neuronal precursor proliferation in the cerebellum by Sonic Hedgehog. Neuron 1999; 22:103-114.

22 Corcoran RB, Scott MP. Oxysterols stimulate Sonic hedgehog signal transduction and proliferation of medulloblastoma cells. Proc Natl Acad Sci USA 2006; 103:8408-8413.

23 Lipinski RJ, Gipp JJ, Zhang J, Doles JD, Bushman W. Unique and complimentary activities of the Gli transcription factors in Hedgehog signaling. Exp Cell Res 2006; 312:1925-1938.

24 Takahashi K, Yamanaka S. Induction of pluripotent stem cells from mouse embryonic and adult fibroblast cultures by defined factors. Cell 2006; 126:663-676.

25 Chen J, Liu J, Yang J, et al. BMPs functionally replace Klf4 and support efficient reprogramming of mouse fibroblasts by Oct4 alone. Cell Res 2011; 21:205-212.

26 Li Y, Zhang Q, Yin X, et al. Generation of iPSCs from mouse fibroblasts with a single gene, Oct4, and small molecules. Cell Res 2011; 21:196-204.

27 Yuan X, Wan H, Zhao X, Zhu S, Zhou Q, Ding S. Combined chemical treatment enables Oct4-induced reprogramming from mouse embryonic fibroblasts. Stem Cells 2011; 29:549553.

28 Shao L, Feng W, Sun Y et al. Generation of iPS cells using defined factors linked via the self-cleaving $2 \mathrm{~A}$ sequences in a single open reading frame. Cell Res 2009; 19:296-306.

(Supplementary information is linked to the online version of the paper on the Cell Research website.) 